\title{
The Research of Students' Comprehensive Quality Evaluation System
}

\author{
Haichang Yin ${ }^{1,}$, Cheng Liü, b \\ ${ }^{1}$ Guangdong Polytechnic of Science and Technology, Zhuhai 519090, China \\ 2Information Engineering University, Zhengzhou 450001, China. \\ ayhc1014@163.com, bliucheng8256@126.com
}

\begin{abstract}
Students' comprehensive quality evaluation, is the important content for transformation of education and talent cultivation. This paper based on the training mode and the evaluation system of West Point, through the expert investigation method, a wide range of questionnaire investigation, establishing Students' comprehensive quality evaluation system, and using the method of fuzzy mathematics evaluation for Students' quality, working for the professional information construction personnel training to provide feedback.
\end{abstract}

Keywords: comprehensive quality; expert investigation; fuzzy mathematics.

\section{Introduction}

In order to improve the comprehensive quality of primary command talent education system gradually established, to establish to adapt to the teaching reform of Students' comprehensive quality evaluation system is particularly important.

\subsection{West point Students leadership training and education system}

The "Students leadership training system" of West point rule the students training draft during the four years learning and training in school, disassemble it as Career anchors, professional behavior, Team spirit, Influence others, Caring for others, Professional ethics, Organize, delegate, Monitor ability, Train subordinates, Decision-making ability and Expression ability, total 12 kinds, for each ability, there are same clear and observable behavior criteria; based on this, establish "Students' 12 kinds of leadership criteria" which is more operational stronger, give the scientific gist for students leadership ability evaluation[1].

\subsection{Current situation of Students' comprehensive quality evaluation in our school}

For Students quality evaluation, our school established "graduate students physical skill examination Detail rules" and "Outstanding students selection criteria", evaluate students" scientific culture and other abilities.

(1)During the process of students evaluation, we should pay more attention on students' daily performance, evaluate students" tasks by corresponding "evaluation form for completing mission".

(2)During the process of students evaluation, we should invite students' leaders, teachers and classmates who are familiar with the students, give evaluation from different ways.

(3)Should set up feedback system for students evaluation, feedback the lack of students during evaluation to students timely, formulate corresponding measure to improve lack. So we can improve effect by evaluation.

\section{Analysis}

According to our requirement for future commander, setting up Students' comprehensive quality evaluation system must include professional base quality, scientific culture quality, leadership ability and psychological quality four first index. According to the requirement of commander quality, there are some second index [2].

During the process of surveying date, using the logarithm of the weighted average method for data conversion, to make sure the weight of each index.

$$
\bar{A}=\frac{\left(A_{1}+A_{2}+\cdots+A_{20}\right)}{20}, \bar{B}=\frac{\left(B_{1}+B_{2}+\cdots+B_{20}\right)}{20}, \bar{I}=\frac{\left(I_{1}+I_{2}+\cdots+I_{20}\right)}{20}
$$


Because the full mark during the process of surveying date is 5, the average date will less than 5 . Now using 5 as the bottom, do logarithm operation for each average date, we can get the $\operatorname{logarithma}=\log _{5} \bar{A}, \mathrm{~b}=\log _{5} \bar{B}, \ldots \mathrm{i}=\log _{5} \bar{I}$, , combine them to get $\mathrm{S}$. Finally, get the weighted average as $r_{i j}=\frac{x_{i j}-\hat{j}_{j} x_{i j}}{\underset{j}{\vee} x_{i j}-\hat{j}_{j} x_{i j}}$.delete the item that weight is less 0.06 , get the statistical result as below table 1 .

Table 1. Expert survey result analysis

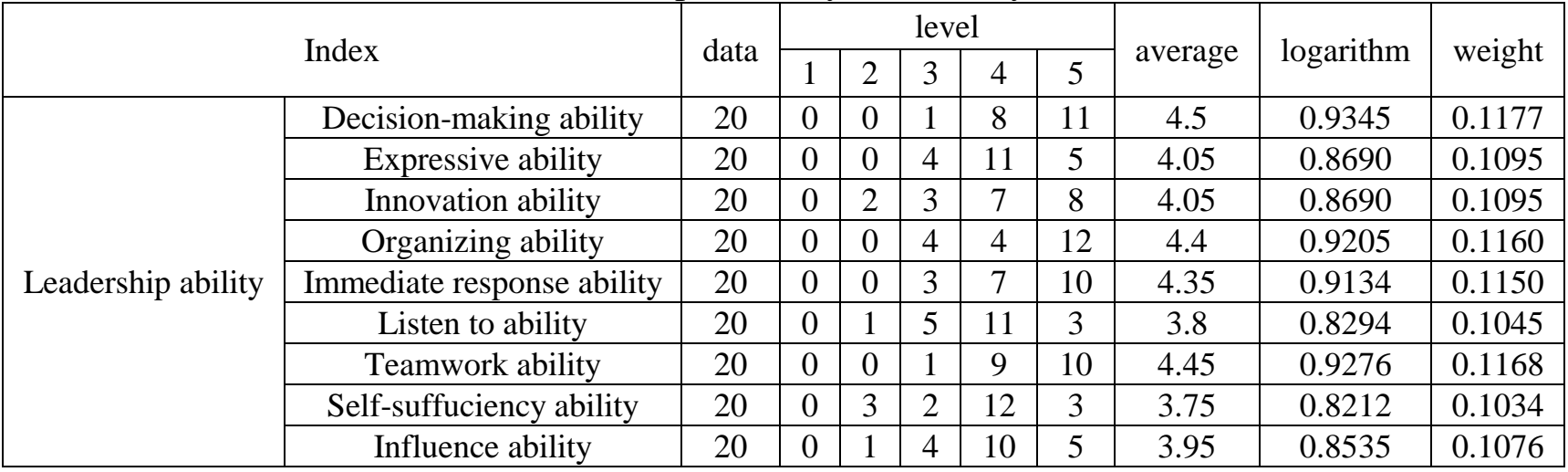

Then we'll get the weight of each index of students' comprehensive quality evaluation system.

\section{Modeling}

\subsection{Establish fuzzy mathematics evaluation model}

Table 2 statistics for the results of the survey of some students' ability of leadership, the leadership of primary index of $\mathrm{U} 1$ evaluation index set for $\mathrm{U} 1=\{\mathrm{U} 11, \mathrm{U} 12, \mathrm{U} 19\}$, contains nine secondary indexes. Setting evaluation grade $\mathrm{V}_{1}=\left\{\mathrm{V}_{11}, \mathrm{~V}_{12}, \mathrm{~V}_{13}, \mathrm{~V}_{14}\right\}=\{\mathrm{D}, \mathrm{C}, \mathrm{B}, \mathrm{A}\}$, four grades. If gets 90-100 equal to A, 80-90 equal to B, 70-80 equal to $\mathrm{C}, 60-70$ equal to $\mathrm{D}$.

Table 2.cadet leadership survey analysis result

\begin{tabular}{|c|c|c|c|c|c|c|}
\hline \multirow{2}{*}{\multicolumn{2}{|c|}{ Indexes }} & \multirow{2}{*}{$\begin{array}{l}\text { Valid } \\
\text { data }\end{array}$} & \multicolumn{4}{|c|}{ percentage } \\
\hline & & & $\mathrm{D}$ & $\mathrm{C}$ & $\mathrm{B}$ & $\mathrm{A}$ \\
\hline \multirow{6}{*}{$\begin{array}{c}\text { Leadership } \\
\text { ability } U_{1}\end{array}$} & Decision-making ability $\mathrm{U}_{11}$ & 10 & 0 & 2 & 5 & 3 \\
\hline & Expressive ability $\mathrm{U}_{12}$ & 10 & 1 & 4 & 3 & 2 \\
\hline & Innovation ability $\mathrm{U}_{13}$ & 10 & 2 & 5 & 2 & 1 \\
\hline & & & & & & \\
\hline & Self-suffuciency ability $\mathrm{U}_{18}$ & 10 & 1 & 3 & 5 & 1 \\
\hline & Influence ability $\mathrm{U}_{19}$ & 10 & 0 & 1 & 8 & 1 \\
\hline
\end{tabular}

At First, evaluates the single index $\mathrm{U}_{1 \mathrm{i}}$, then determining the membership of index of evaluation grades from $U_{1 i} V_{1 j}(j=1,2,3,4)$ membership $R_{i j}$, get the set of indicators to evaluate $R_{i j} I=\left\{R_{i 1} R_{i 2}\right.$ $\mathrm{R}_{\mathrm{i} 3} \mathrm{R}_{\mathrm{i} 4}$ \}, shows the single index evaluation of $U_{1}$ from $i$. Then we can constitute a fuzzy evaluation matrix:

$$
R_{1}=\left(\begin{array}{ccc}
R_{11} & \cdots & R_{14} \\
\vdots & \ddots & \vdots \\
R_{91} & \cdots & R_{94}
\end{array}\right)
$$

To overcome the homogenization, using the optimal formula (the bigger one) transformed the eigenvector matrix $\mathrm{R}_{1}$, get the relative membership degree matrix:

$$
\tilde{R}_{1}=\left(\begin{array}{ccc}
\tilde{R}_{11} & \cdots & \tilde{R}_{14} \\
\vdots & \ddots & \vdots \\
\tilde{R}_{91} & \cdots & \tilde{R}_{94}
\end{array}\right)
$$

Relative membership degree is:

$$
r_{i j}=\frac{x_{i j}}{\underset{j}{\vee} x_{i j}+\hat{j}_{j} x_{i j}}
$$


or $r_{i j}=\frac{x_{i j}-\hat{j}_{j} x_{i j}}{\underset{j}{\vee} x_{i j}-\hat{j}_{j} x_{i j}}$

$r_{i j}$ Describes the relative membership degree of the $\mathrm{j}$ to $\mathrm{i}, \underset{j}{\vee} x_{i j}$ is the $\max , \underset{j}{\wedge} x_{i j}$ is the min, which are both eigenvalues of $i$, with $j=1,2 \ldots n$.

The fuzzy optimum selection model like:

$u_{j}=\frac{1}{1+\left\{\frac{\sum_{i=1}^{m}\left(\omega_{i}\left(g_{i}-r_{i j}\right)\right)^{p}}{\sum_{i=1}^{m}\left(\omega_{i}\left(r_{i j}-b_{i}\right)\right)^{p}}\right\}^{\frac{2}{p}}} \quad j=1,2, \cdots, n$

$\mathrm{P}$ is the distance parameter, which is Hamming distance when equals to 1, Euclidean distance by 2.

\subsection{Fuzzy comprehensive evaluation}

We can get the fuzzy matrix of the evaluation grades of $U_{1}$.

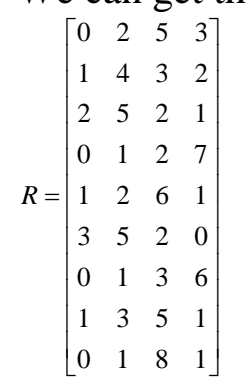

From the matrix we can get:

$$
\begin{aligned}
& \mathrm{g}_{1}=\left(\begin{array}{lllllllll}
1 & 0.8 & 0.83 & 1 & 0.86 & 1 & 1 & 0.83 & 1
\end{array}\right)^{\mathrm{T}} \\
& \mathrm{b}_{1}=\left(\begin{array}{lllllllll}
0 & 0.2 & 0.17 & 0 & 0.14 & 0 & 0 & 0.17 & 0
\end{array}\right)^{\mathrm{T}}
\end{aligned}
$$

According to the leadership of each index weight, we can get:

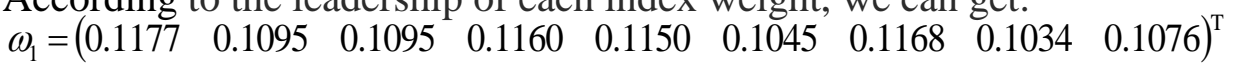

By using formula (6) and make p equals to 1 , we can get:

$\mu_{1}=\left(\begin{array}{llll}0.0025 & 0.209 & 0.8043 & 0.3038\end{array}\right)$

The evaluation matrix normalized processing, then:

$$
\mu_{1}=\left(\begin{array}{llll}
0.0019 & 0.1583 & 0.6093 & 0.230
\end{array}\right)
$$

Then we can get the leadership comprehensive evaluation value of $\mathrm{U}_{1}=85.64$.

According to the evaluation procedure, the basis for professional quality, scientific quality and psychological quality, we can get the data from others and the inspection results of students, then establishing the corresponding eigenvector matrix, using the optimal relative formula, converts it to optimal relative matrix, the calculation using the fuzzy optimization model, then the results and evaluation rating score vector multiplication, finally get the indexes of evaluation results [3].

\section{Summary}

Modelling students' comprehensive quality evaluation system, can students comprehensive test and evaluation of the performance. At the same time, using expert questionnaire method and fuzzy mathematics method to evaluate, using a combination of qualitative and quantitative analysis, more scientific and objective, can make students realize themselves, focus on foster strengths and circumvent weaknesses. The managements of the university can find the cultivating method through the feedback information, according to their aptitude, to carry out special training plan of the students' individual. 


\section{References}

[1]. Haifu Xu, The cultivation of the students' ability of leadership and inspiration of West point., Journal of Higher Education Research ,the fourth issue of volume 25, 2002

[2]. Shaoxiong Chen, The integrated use of analytic hierarchy process (ahp) and fuzzy mathematics method to evaluate the graduate cultivation model in our country. Higher Education Exploration, the 3 rd issue , 2005

[3]. Anmin Luo, "Academic education HeXun", the research of management methods of the students' leadership ability. 2004 